\title{
Energy Consumption Analyzing in Single hop Transmission and Multi-hop Transmission for using Wireless Sensor Networks
}

\author{
Ekhlas K. Hamza* Heba H. Alhayani** \\ ***Department of Control and Systems Engineering / University of Technology/ Baghdad \\ * Email: Ekhlas_kadhum@yahoo.com \\ ** Email: heba_alhayani@yahoo.com
}

(Received 11 April 2017; accepted 31 October 2017)

https://doi.org/10.22153/kej.2018.10.009

\begin{abstract}
Wireless sensor networks (WSNs) are emerging in various application like military, area monitoring, health monitoring, industry monitoring and many more. The challenges of the successful WSN application are the energy consumption problem. since the small, portable batteries integrated into the sensor chips cannot be re-charged easily from an economical point of view. This work focusses on prolonging the network lifetime of WSNs by reducing and balancing energy consumption during routing process from hop number point of view. In this paper, performance simulation was done between two types of protocols LEACH that uses single hop path and MODLEACH that uses multi hop path by using Intel Care i3 CPU $(2.13 \mathrm{GHz})$ laptop with MATLAB (R2014a). The simulation results showed how the multi-hop protocol was more energy efficient than single hop protocol.
\end{abstract}

Keywords : Energy-efficient, LEACH, MODLEACH, Network-lifetime, Wireless sensor network

\section{Introduction}

The improvements in MEMS (Micro ElectroMechanical Systems) as well as in wireless communiqué have boost the extending of billions of small and low cost wireless strategies as well as various type of wireless systems which connect these devices with or deprived of any present arrangement[1] .

Wireless Device Networks (WSNs) are dividing data acquisition system consisting of plentiful Wireless Sensor Nodes. The fast arrangement, self- group and fault tolerance characteristics of WSNs make them a very promising sensing technique for environmental, military and health applications. A WSNs consists of hundreds and thousands off Sensor nodes (SNs). These SNs have ability to monitor phenomenon's in the environment such as temperature, sound, pressure and communicate this information wirelessly with each other or directly to the base station (BS) [2].SNs consists of sensors, processor, memory, communication system, mobilizer, position finding system, and power units, see fig 1 [3].

One of the challenges of the successful WSNs application is the energy consumption problem. [4] SNs have incomplete battery lifetime. Frequently their battery cannot be exchanged and re-energized due to area of their deployment, so, the network life span be contingent upon the initial battery capacity of SNs. A careful management of the resources should be used here to increase lifespan of WSNs. And the Excellence of routing proprieties also be contingent upon the amount of data (Real Facts Indication) positively expected by BS from SNs organized in the network area. Number of routing protocols has been proposed for WSNs [5, 6], figure 2 show the routing protocol classification.

Proprieties are more categorized as practical, sensitive and mix, conditional on the kind of 
message routes processed within the network for data communication from the source to BS .

In Active routing proprieties all the pathways are considered before the BS makes an beginning to connect with the SNs in the system, whereas in Reactive routing protocols the path values are calculated only when required. the path values are calculated and best path is choice for data transmission when a BS wants to communicate with SNs. Hybrid routing protocols is a gathering of both proactive and reactive protocols, depending on the type of communication the path calculated from the BS to the source[7]

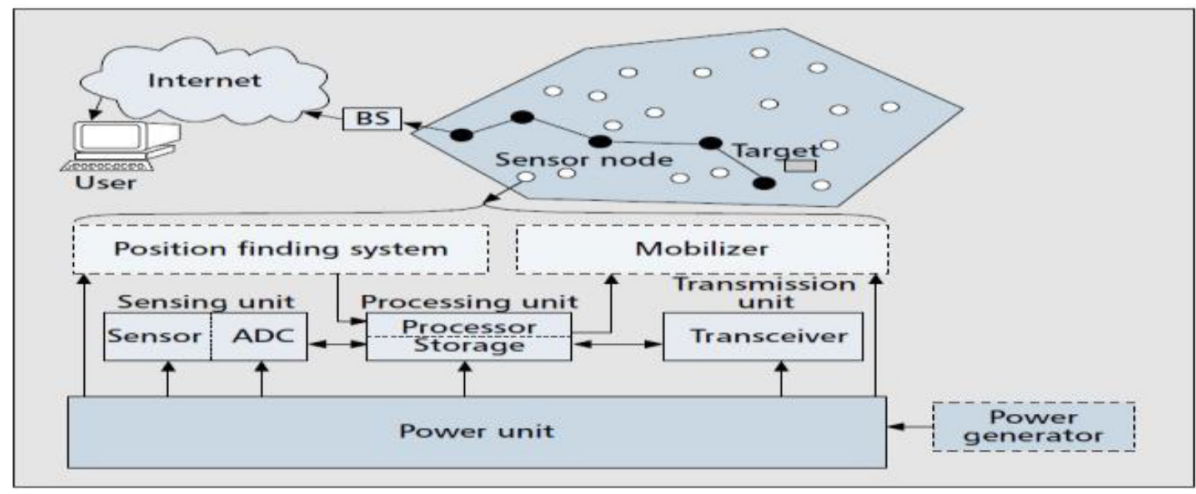

Fig. 1. Components of WSNs Node[3].

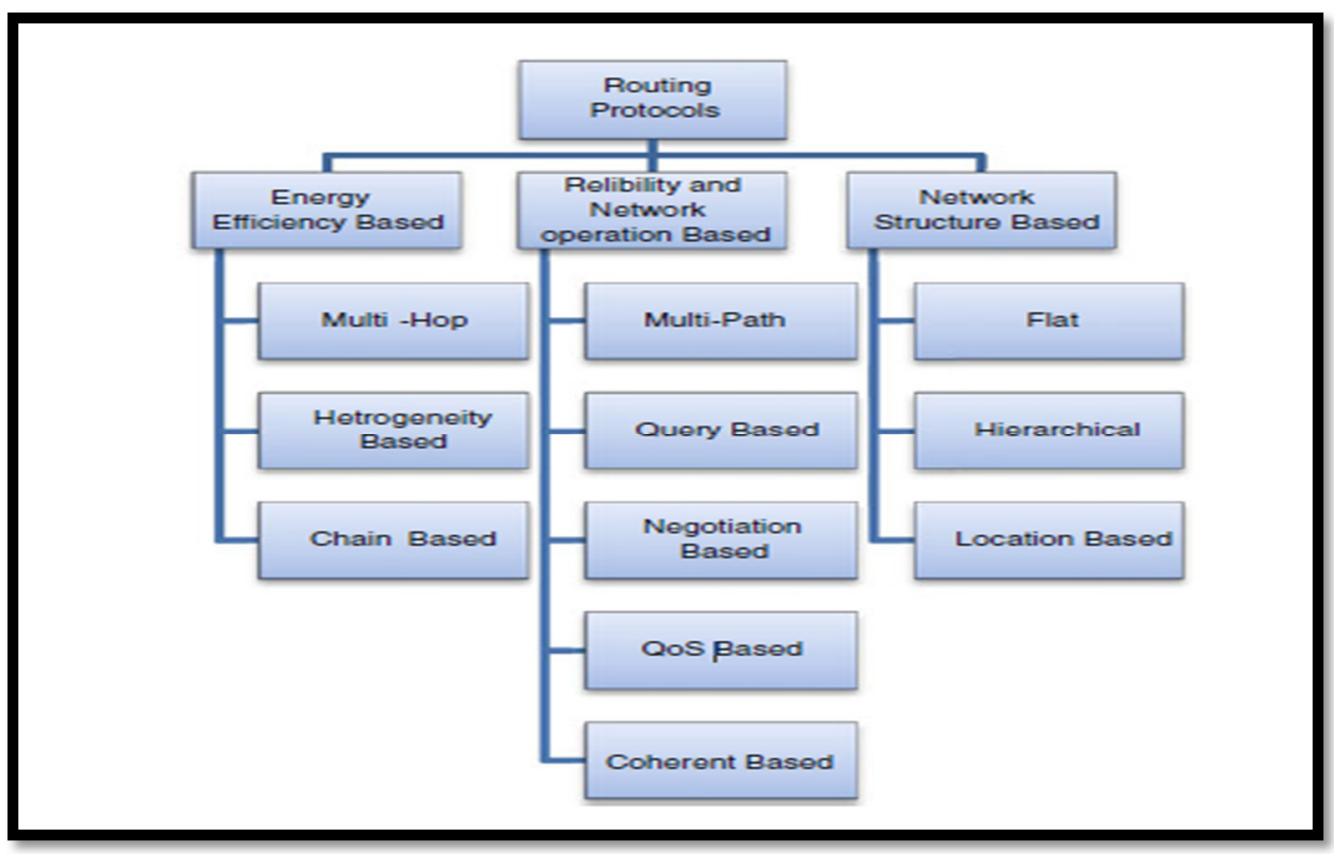

Fig. 2. General classification of Routing protocols in WSNs[6, 8].

Data gathering technique, also known as data fusion, is to optimize transmit data and obtain energy efficiency. Data collection is defined as the collection of information from several SNs in a systematic manner and sent to the BS for processing. Since SNs could make important dismissed information, that could be similar data aggregated as well for achieving the reducing of data in the small groups of significant material While, Data combination is the combination of data that minimized into small groups of meaningful information from various sources according to a certain aggregation function $[9,10]$

Single hop transmission using when small scale network and when SNs close to BS. multi hop transmission using when large scale network and when SNs far away from BS . When the transmitter distance is proportional to d4 the long distance is divided into sub - 
distances this needs more energy efficient, the power attenuation is proportional to the $\mathrm{d} 2$ [1] .

This research is planned as follows: Section 2 consists of the brief description about the Low Energy Adaptive Clustering Hierarchy $(\mathrm{LEACH})$ and Modify Low Energy Adaptive Clustering Hierarchy (MOD-LEACH) protocols. Section 3 describes System Model and Assumptions. Section 4 describes the implementation of the experiment Section 5 simulation results along with comparisons are discussed and lastly, section 6 concludes the paper.

\section{Low Energy Adaptive}

\subsection{Clustering Hierarchy (LEACH)}

The first hierarchical routing protocol in the WSNs is LEACH (Low-Energy Adaptive Clustering Hierarchy) protocol[11, 12] , single hops path selection[13] and Proactive routing protocol[14] .

LEACH protocol is organize the SNs into cluster, there is elected SNs called cluster head $(\mathrm{CH})$ and the rest nodes a called cluster member (CM) (LEACH shown in figure 3 ). LEACH down into rounds, each round has two phase :the setup phase and the steady - state phase .

\subsubsection{Setup Phase}

In this phase, clusters are formed and a $\mathrm{CH}$ is chosen for each cluster. Every $\mathrm{SN}$ (n) chooses number randomly between 0 and 1 . If this unsystematic quantity is less than inception value $T(n)$, the $\mathrm{SN}$ will become a $\mathrm{CH}$ for the present round[15].

$\mathrm{T}(\mathrm{n})$ is defined as follows:

$\mathrm{T}(\mathrm{n})=\frac{p}{1-p\left(r \bmod \left(\frac{1}{p}\right)\right)} \quad$ if $\mathrm{n} \in \mathrm{G}$

0 otherwise

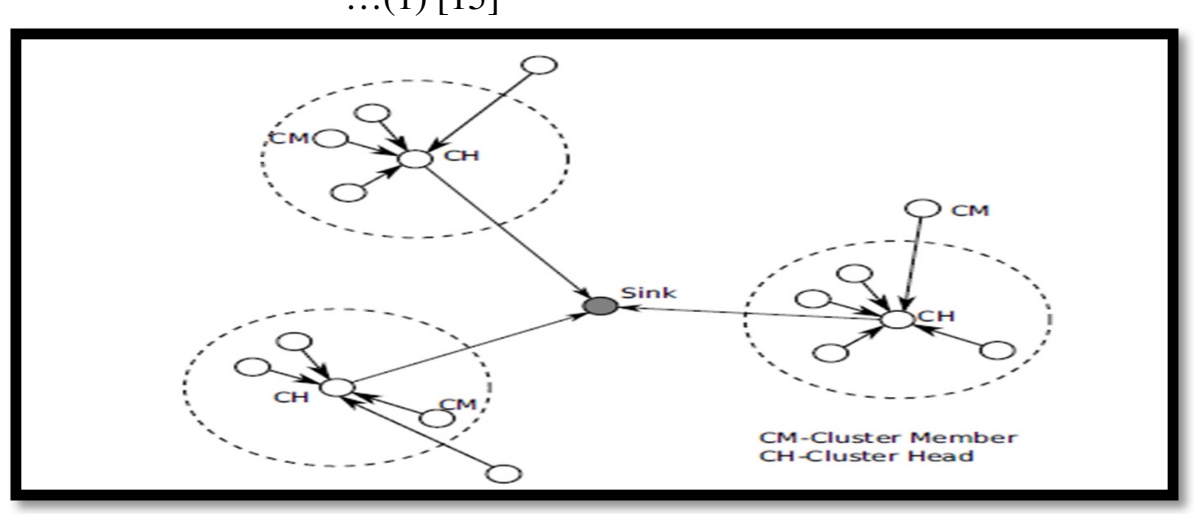

Where

- $p$ is the percent. $\mathrm{CH}$ to all of the nodes $-\mathrm{r}$ is the current round

- $G$ is the set of nodes that was elected in the past $1 / p$ rounds of election.

$-\bmod ()$ is the modulus function.

When $\mathrm{r}=0$, the chance of each $\mathrm{SN}$ can become the $\mathrm{CH}$ is $\mathrm{P}$. In the first $\mathrm{r}$ rounds if and $\mathrm{SN}$ becomes the $\mathrm{CH}$, it can be not reelected in the future time $(1 / \mathrm{P}-\mathrm{r})$ round which boost the option of other SNs to develop a CH. All SNs have the possibility of $\mathrm{P}$ to be a $\mathrm{CH}$ once again over and over again when 1/P rounds. A SN is designated as a $\mathrm{CH}$ at random. The $\mathrm{CH}$ node broadcasts messages to the environment, and other SNs select a cluster to join in, subject to the intensity of the broadcasted messages they received, and then inform the corresponding $\mathrm{CHs}$. A CH communicates with a BS directly and the CMs only communicate with the $\mathrm{CH}$ in their own cluster. While clusters are forming, each $\mathrm{CH}$ creates a TDMA schedule giving to the number of SNs in the cluster. Each SN transfers their detected data to its $\mathrm{CH}$ through its distributed transmission time in the TDMA [11-13].

\subsubsection{Steady-State Phase}

In the phase, all the CMs start detecting data and send it to their $\mathrm{CHs}$ giving to the TDMA schedule. The $\mathrm{CH}$ compresses the receiving data and sending it to the BS. Communication will be via direct-sequence spread spectrum; each cluster uses a unique spreading code to reduce inter-cluster interference. After an estimated period of time the network again goes into the setup phase and enters another round of selecting $\mathrm{CHs}[11-13]$.

Fig. 3. LEACH protocol [16] 


\subsection{Modify Low Energy Adaptive Clustering Hierarchy (MOD-LEACH)}

MOD-LEACH is one of hierarchical routing protocols in the WSNs introduce by [14], work of this protocol based on LEACH. It is multi hop protocol if the distance between $\mathrm{CHs}$ and $\mathrm{BS}$ far multi hop path communication is adopted among CHs [8] and Proactive routing protocol [14].

This protocol modulating LEACH through put in "efficient $\mathrm{CH}$ replacements scheme". For the next round, it has the threshold in the $\mathrm{CH}$ formation.

The existing cluster will also remain $\mathrm{CH}$ for the next round when it has no enough energy to consume during its time and has more energy than essential edge .This matter describes how energy unused in routing packets for cluster formation and new $\mathrm{CH}$ can be saved accordingly. So, if $\mathrm{CH}$ has low energy than required for threshold; then LEACH algorithm will be exchange In cluster formation for limiting energy usage, according transmission nature two different levels can be used of power in order to amplify signals .Three methods of communication in one cluster based system.

1)Intra Cluster Transmission deals CMs sense data and explosion recognized information to $\mathrm{CH}$, 2) Inter Cluster Communication can be named for the transmission/ reception between two $\mathrm{CHs}, 3$ ) $\mathrm{CH}$ To BS Transmission is the transmission while a CH transmitting it's data straight to BS[14].

MOD-LEACH is more enhanced by using the idea of soft and hard threshold as presented by TEEN[14].

\section{System Model and Assumptions 3.1. Assumptions}

The following expectations are made for the new scheme:

- All SN are homogenous and stationary after deployment

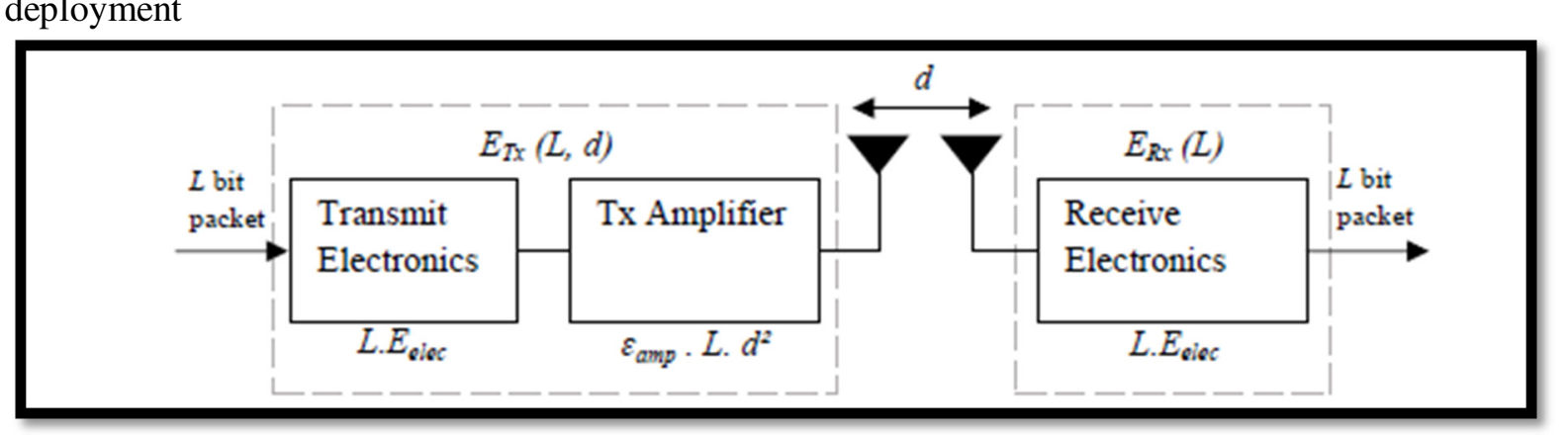

Fig. 4. First order energy model[17]
- The sink stationary and has the information about the location of each node.

- Each SN makes one data package per time unit to be communicated into sinks. we denote to each time unit as a round for simplicity.

- SNs are dispersed in a 2-dimensional space and cannot be recharged after deployment.

- Each SN is assigned a unique identifier (ID).

- Each SN has the same initial power.

- Energy of transmission depends on the distances (source to destination) and data sizes.

- Radio's channel can be considered as a symmetric i.e. to communicate a message from node $\mathrm{M}$ to node $\mathrm{N}$ the energy should be required and verse versa if a message from node $\mathrm{N}$ to node $\mathrm{M}$ is transmitted for a given SNR.

- the SN has no cooperation, moreover; it doesn't attempt to contact the channel instantaneously and we study a lined wireless network designed for this examination.

\subsection{Radio Energy Model}

For approximation the energy depletion for SNs and the system lifetime, we usage the principal instruction energy model (shown in fig. 4).

This model introduces (presents) the energy consumed for sending and receiving L-bit communication above a distance $d$ then transferring interested in a wireless account transmission and multi pathway transmission simulations. The communication energy in wireless typical can be considered as a relational to space $d^{2}$, while for multi pathway spread classic, the energy is relational to space $d^{4}$ according to unlike pathways that transfers the communicated indication to extent the receiver itself.[17]. 
Energy sends information of $L$ bit package above a space $d$ from a node to a $\mathrm{CH}$ otherwise a $\mathrm{BS}$ which is designed giving to under stated equation (2).

$\mathrm{E}_{\mathrm{Tx}}=\mathrm{E}_{\text {elec }}(\mathrm{L})+\varepsilon_{\mathrm{amp}}(\mathrm{L}, \mathrm{d})$

Where

- Eelec energy consumed to transmit or receive the signals electronic circuit

- Eamp energy consumed to transfer at a longer distance by the amplifier

- L No. of bits in a packet

- D Distance between transmitter and receiver Obtaining the crossover distance do that describes the spread evolution from through pathway to multipath typical:

$\mathrm{do}=\sqrt{\frac{\varepsilon f s}{\varepsilon m p}}$

where

- Efs Energy consumed to communicate at a smaller distance by the amplifier

- Eamp energy spent to communicate at a longer distance by the amplifier

The energy disbursed for wireless spread (where d $<$ do) ETx-fs is defined by:

$\mathrm{E}_{\mathrm{Tx} \text {-fs }}=\mathrm{E}_{\text {elec}} \cdot \mathrm{L}+\varepsilon_{\mathrm{fs}} \cdot \mathrm{L} \cdot \mathrm{d}^{2}$

Where

- Eelec energy depleted in electronic path to communicate or accept the indication

- Efs Energy consumed by the amplifier to communicate at a shorter distance

- L No. of bits in a packet

- D Distance between transmitter and receiver

The energy spent for multi-path spread (where $\mathrm{d} \geq$ do) ETx-mp is given by:

$\mathrm{E}_{\mathrm{Tx}-\mathrm{mp}}(\mathrm{L}, \mathrm{d})=\mathrm{E}_{\mathrm{elec}} \cdot \mathrm{L}+\varepsilon_{\mathrm{mp}} \cdot \mathrm{L} \cdot \mathrm{d}^{4}$

Where

- E elec energy spent in electronic circuit to transmit or receive the signal

- Eamp energy consumed by the amplifier to transmit at a longer distance

- L No. of bits in a packet

- D Distance between transmitter and receiver The energy expended to receive $L$-bit message is defined as [11]:

$\mathrm{E}_{\mathrm{Rx}}(\mathrm{L})=\mathrm{E}_{\text {elec. }} \mathrm{L}$

Where

- Eelec Energy spent in electronic path to communicate or accept the indication

- L No. of bits in a packet

\section{Simulation}

To evaluate the performance of single hop and multi hop in WSNs, we have performed in MATLAB (R2014a) when run for 5000 rounds with randomly deployment nodes. Simulation parameters shown in table 1.

Single hop in our work can be obtained by implementation the LEACH and the MODLEACH protocol present in implemented to obtain the multi hop in WSN, both LEACH and MODLEACH are proactive (periodical transmissions).

Our goals in conducting the simulation are as compare the performance of the single hop protocol and multi hop protocol in WSNs on the foundation of energy intemperance and the lifetime of the system.

Table 1,

Simulation parameter

\begin{tabular}{ll}
\hline Parameter & \multicolumn{1}{c}{ Value } \\
\hline Network size & $100 \mathrm{~m} \times 100 \mathrm{~m}$ \\
Base station position & $50 \mathrm{~m} \times 75 \mathrm{~m}$ \\
Number of nodes & 100 \\
Percentage of CH & 0.1 \\
Message size & $2000 \mathrm{bit}$ \\
Communication and reception & $50 \mathrm{~nJ} / \mathrm{bit}$ \\
energy & \\
Energy increase for wierless & $10 \mathrm{pJ} / \mathrm{bit} / \mathrm{m}^{2}$ \\
Energy amplification for & 0.0013 \\
multi path & $\mathrm{pJ} / \mathrm{bit} / \mathrm{m}^{2}$ \\
initial energy for nodes & $0.5 \mathrm{~J}$ \\
Data aggregation energy & $5 \mathrm{~nJ} / \mathrm{bit} / \mathrm{message}$ \\
\hline
\end{tabular}

\section{Experiments}

In this work use two metrics to analyze and compare the performance of the protocols. They are:

Normal energy degenerate: This metric indications the average intemperance of energy per SNs over time in the network. Three features are measured to be accountable for intense the energy throughout facts routing which are: data send, data receive and data combined.

Network lifetime: is the time from the early of the test (turning all sensors on) until the instant when the first one expired. We use term FND (first node dead), HND (half node dead ) and LND(last node dead ) for indicate the lifetime of network.

\section{Results}

\subsection{Average Energy Dissipated}

Normal remaining energy of system per round as in shows fig. 5. Each node has $0.5 \mathrm{~J}$.The total 
energy for 100 node is $50 \mathrm{~J}(0.5 \mathrm{~J} * 100=50 \mathrm{~J})$. Fig 5 clearly depicts that multi hop outperforms single hop in positions of energy depletion per round. Multi hop yields minimum energy consumption than single hop because the space among SNs is measured and wireless spread is frequently adopted as the minimization of inter-nodes space outcome in $d 2$, as the connection cost factor in its place of $d 4$ for multipath spread. This spread typical transformation well rises the energy productivity of the system.

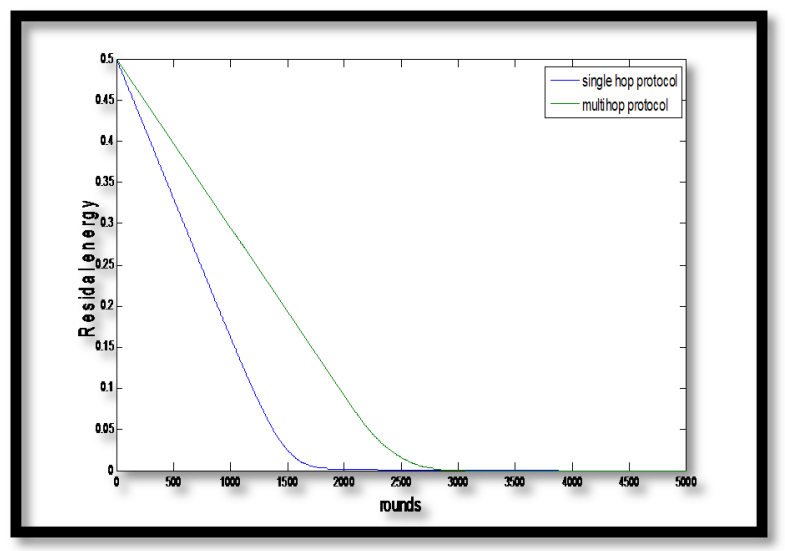

Fig. 5.Complete energy of the system against broadcast rounds for single hop and multi hop protocols.

\subsection{Total Number of Nodes Dead:}

Fig $6 \&$ table 2 shows dead node number of network per round .The FND is dead of the single hop protocol after 762 rounds and the LND after 2650 rounds. The FND of multi hop protocol is dead after 2020 rounds and LND is lifeless after 3064 rounds. But totally the instrument nodes are expired when 2650 rounds for single hop procedure, wherever at the identical round practically $20 \%$ of nodes are still active in the multi hop .Accordingly, the achieved instrument system by the multi-hop remains alive during more rounds.

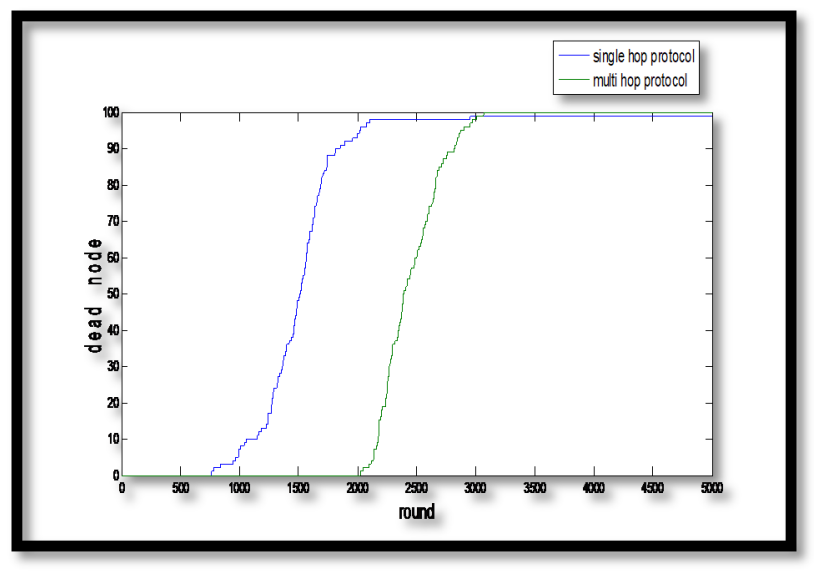

Fig. 6. Total dead node of the system against transmission rounds for single hop and multi hop protocols

Table,

Dead nodes for Single hop and Multi hop protocols

\begin{tabular}{llll}
\hline & FND & HND & LND \\
\hline Single hop & 762 rounds & 1510 & 2650 \\
& & rounds & rounds \\
Multi hop & 2020 & $\begin{array}{l}2389 \\
\text { rounds }\end{array}$ & $\begin{array}{l}3064 \\
\text { rounds }\end{array}$ \\
\hline
\end{tabular}

\section{Conclusion}

Steering process is of very significance in enhancing energy depletion in WSN. In this research, we present a compare the performance of the single hop protocoland multi hop protocol in WSN on the basis of energy dissipation and the lifetime of the network. Outcomes of achieved models reveal that the multi hop protocol outclasses single hop procedure and allows the SNs to improve communication energy and increasing the network lifetime.

\section{References}

[1] J. Wang, "Hop-based Energy Aware Routing Scheme for Wireless Sensor Networks",Ph.D. thesis , Kyung Hee University Seoul, Korea, February, 2010.

[2] M. Hussain , R. H. Chowdhury, M. Hassan , M. A. Hossain ,S. Jahan and M. H. Rahman , " Performance Analysis of Various Modulation Schemes for Achieving Energy Efficient Communication over Fading Channel for Wireless Sensor Network " IOSR Journal of Electronics and Communication 
Engineering (IOSR-JECE), Vol. 11, Issue 1, PP. 97-100, 2016.

[3]J. N. Al-Karaki and A.E. Kamal, "Routing techniques in wireless sensor networks: a survey". IEEE wireless communications, Vol. 11, No. 6 ,pp. 6-28, 2004.

[4]J. Wang, Y. Niu, J. Cho and S. Lee, " Analysis of Energy Consumption in Direct Transmission and Multi-hop

[5] Transmission for Wireless Sensor Networks ", Third International IEEE Conference on Signal-Image Technologies and InternetBased System , IEEE, 2007.

[6] M. Aslam, M. B. Rasheed, T. Shah, A. Rahim, Z. A. Khan, U. Qasim, M. W. Qasim, A. Hassan, A. Khan and N. Javaid ," Energy optimization and Performance Analysis of Cluster Based

[7] Routing Protocols Extended from LEACH for WSNs", arXiv preprint arXiv:1309.4373, 2013.

[8]P. K. Singh," Energy efficient clustered chain based power aware routing protocol for wireless sensor networks" , Master thesis , National Institute of Technology Rourkela, India , 2013.

[9] M. Ali and S. K. Ravula, " Real-time support and energy efficiency in wireless sensor networks " , Master Thesis , Halmstad University , Halmstad, Sweden, January, 2008.

[10] S. Rani and S.H. Ahmed," Multi-hop routing in wireless sensor networks: An overview, taxonomy, and research challenges"., Springer ,2015.

[11] Jain, T. "Wireless environmental monitoring system (WEMS) using data aggregation in a bidirectional hybrid protocol." in International Conference on Information Systems, Technology and Management, Springer, 2012.

[12] P. Kumar, A. Chaturvedi, and M. Kulkarni, "Geographical location based hierarchical routing strategy for wireless sensor networks". in Devices, Circuits and Systems (ICDCS), 2012 International Conference on,IEEE, 2012.
[13] W.B. Heinzelman, A.P. Chandrakasan, and H. Balakrishnan, "An application specific protocol architecture for wireless microsensor networks", IEEE Transactions on

wirelesscommunications, Vol.1,No.4,PP.660 $-670,2002$.

[14] D. Verma, R. Jain, and A. Shrivastava, "Performance Analysis of LEACH and LEACH-CC Routing Protocol in Wireless Sensor Network" . IUP Journal of Telecommunications ,Vol.8 , No.3 PP.45, 2016.

[15] L. An, A. Li , L. Pang and G. He, " Analyss and Comparison of Routing Protocol of Wireless Sensor Network", International Journal of Online Engineering, Vol.12 ,No.10, 2016.

[16] D. Mahmood, N. Javaid, S. Mahmood, S. Qureshi, A. M. Memon and T. Zaman," MODLEACH: a variant of LEACH for WSNs ", in Broadband and Wireless Computing, Communication and Applications (BWCCA), 2013 Eighth International Conference on.,IEEE ,2013.

[17] W.R., Heinzelman, A. Chandrakasan, and H. Balakrishnan," Energy-efficient communication protocol for wireless microsensor networks", in System sciences, 2000. Proceedings of the 33rd annual Hawaii international conference on,IEEE , 2000.

[18] A.K. Singh, S. Rajoriya, S. Nikhil, and T. K. Jain," Design constraint in single-hop and multi-hop wireless sensor network using different network model architecture", in Computing, Communication \& Automation (ICCCA), 2015 International Conference on. 2015. IEEE.

[19] I.F. Akyildiz and M.C. Vuran, "Wireless sensor networks", John Wiley \& Sons Vol. 4. 2010. 


\title{
تحليل استهلاك الطاقة عند الاتتقال بقفزة واحدة و بقفزات \تعددة لشبكات الاستشعار اللاسلكية
}

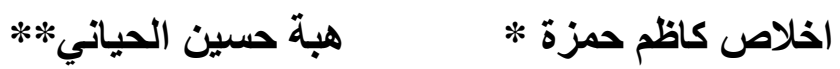

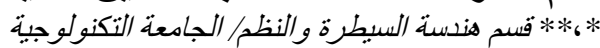

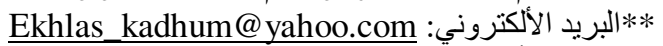 \\ heba_alhayani@yahoo.com البريد الألكتروني:
}

يمكن استخدام شبكات الاستشعار اللاسلكية في العديد من التطبيقات مثل التطبيقات العسكرية و المراقبة و الصحة و الصات الصناعة و وغيرها. من اهم

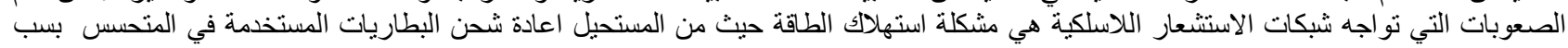

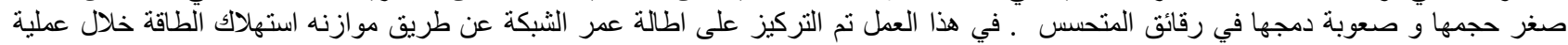

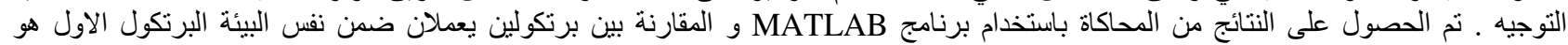

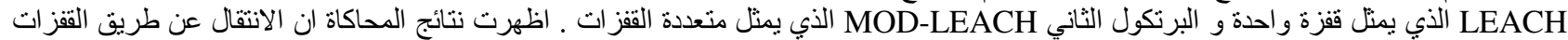
المتعددة افضل من القفزة الو احدة من ناحية استهلاكك الطاقة و الطالة عمر الثبكة . 